\title{
Activismo e investigación: recuperando el concepto de neutralidad valorativa en la formación de tipos ideales
}

\author{
Activism and research: recovering the concept of value neutrality in the \\ formation of ideal types
}

\author{
Antonio Cáñez Cota \\ acanez@conacyt.mx \\ Consorcio de Investigación y Diálogo sobre Gobierno \\ Local, México
}

Cita sugerida: Cáñez Cota, A. (2020). Activismo e investigación: recuperando el concepto de neutralidad valorativa en la formación de tipos ideales. Revista Latinoamericana de Metodología de las Ciencias Sociales, 10(2), e077. https://doi.org/10.24215/18537863e077

Recepción: 04 Febrero 2020

Aprobación: 24 Agosto 2020

Publicación: 01 Diciembre 2020

\begin{abstract}
Resumen: El presente artículo discute la propuesta metodológica weberiana de tipo ideal como una manera de encontrar neutralidad-libertad valorativa en la investigación social contemporánea, la cual exige un mayor involucramiento de los investigadores con los problemas prácticos que ocurren a su alrededor. En la actualidad, el individualismo metodológico es una de las metodologías más utilizadas, su fundación se sostiene en las aportaciones de Max Weber, que se pueden resumir en tres: la primera se refiere a la transparencia de la cuestión valorativa del investigador; la segunda es la construcción de una acción racional de tipo ideal como instrumento de interpretación con alta disciplina científica; por último, se muestra el manejo de la información empírica para imputar una relación causal apropiada para el contexto histórico que se analiza. La principal aportación del artículo es que muestra cada una de las fases del método de elaboración de tipos ideales, con el fin de ofrecer una alternativa para el tránsito entre la investigación y el activismo: separar y explicitar los valores teóricos-conceptuales de los prácticosemocionales.
\end{abstract}

Palabras clave: Tipo ideal, Neutralidad valorativa, Activismo, Max Weber, Individualismo metodológico.

\begin{abstract}
This article discusses the Weberian ideal-type proposal as a way to find value freedom-neutrality in the contemporary social research, which demands greater involvement of researchers with contextual practical problems that occur around them. Currently, methodological individualism is one of the most used methodologies and it is necessary for social researchers to know the bases and assumptions that founded this tradition. Its foundation is based on the contributions of Max Weber, which can be summarized in three: the first refers to the transparency of the value question of the researcher; the second contribution is the construction of an ideal-type rational action as an instrument of interpretation with high scientific discipline; finally, it is shown the management of empirical information to impute a causal relationship for the historical context analyzed. Th main contribution of the article is that it shows each phase of the ideal type method, in order to offer an alternative for the transit between research and activism: separating the theoretical-conceptual values of the practical-emotional ones.
\end{abstract}

Keywords: Ideal type, Value neutrality, Activism, Max Weber, Methodological individualism. 


\section{INTRODUCCIÓN}

El ejercicio de las ciencias sociales enfrenta hoy a distintos paradigmas que disputan la superioridad de ser la mejor perspectiva para explicar la realidad y acercarse a la verdad, mas nunca a la "Verdad". Estos debates teóricos llevan cientos de años, entre ellos destacan las discusiones entre cuantitativos y cualitativos, así como entre las ciencias físico-naturales (también denominadas como "duras") y las ciencias socialeshumanidades. Al interior de estas batallas, también existen las disputas intradisciplinarias por la validación de sus paradigmas frente a otras formas de ver el mundo. Una de las últimas polémicas que han generado debate en las ciencias sociales es la del investigador como activista social discutida por diversos investigadores, entre los que se encuentran Raymond Aron y Jean Paul-Sartre. Por un lado, quienes defienden la idea de la investigación-activismo argumentan que las ciencias sociales deben ser aplicadas a la solución de problemas sociales urgentes. Por otra parte, los que defienden la idea de investigación-apartidista argumentan que el activismo puede nublar el juicio objetivo de la investigación científica. Estas posiciones han producido y siguen produciendo apasionadas discusiones en torno a quién tiene una mejor perspectiva o paradigma científico para entender la realidad y, por ende, responder a sus necesidades.

Estas diferencias se acentúan cuando algunos académicos tienden a menospreciar los enfoques teóricos que van en contra de sus ideologías-perspectivas, ya que consideran que la manera de ver el mundo de otros es inferior a la de ellos. Las personas de mente dogmática no escuchan, no son receptivas, sino que se encuentran a la defensiva para contraatacar cualquier argumento, sin meditar previamente acerca de la situación que se le presenta o incluso reconocer que en ocasiones no se tiene la certeza de una respuesta con evidencia. Cuando se presume la propia ideología, valores y creencias, el investigador social es atrapado por la vanidad intelectual y el desgaste que implica querer tener siempre la razón, por lo que no puede haber pensamiento flexible sin humildad. Esta sencillez se nutre de la autocrítica, donde el investigador tiene plena consciencia de sus limitaciones y presenta una actitud crítica en el sentido de dudar de toda afirmación, sospechar de los argumentos e ideas que se reciben, e incluso dudar de los propios pensamientos (Riso, 1951).

El hecho de que un académico sea activista social no representa problema alguno para la calidad de la investigación científica, siempre y cuando no mezcle los valores prácticos del activismo con los valores teóricos de las distintas explicaciones a los fenómenos sociales; es decir: estos dos roles deben estar separados (Weber, 1997). Ser crítico es tomar una posición ideológico-valorativa, tener la mente abierta para buscar información empírica que respalde o contradiga las ideas preconcebidas y dudar de la validez y temporalidad de las mismas. Algunos investigadores consideran que ser crítico es señalar constantemente que las cosas están mal con argumentos cíclicos y cerrados, como los que marcan la existencia de un sistema opresor. Argumentos que pueden ser plausibles cuando se establecen las variables específicas por las que dicho sistema falla.

Estas disputas entre paradigmas de investigación se encuentran en el contexto de la discusión acerca de la objetividad de las ciencias sociales, donde uno de los puntos más álgidos es la relación del rol de los valores del investigador con el análisis del objeto de estudio; mientras para unos es necesario que el investigador no mezcle sus valores personales en la investigación de la acción social, para otros esto es imposible de alcanzar. El estudio de la acción social ha sido tratado de manera diferente por los grandes pensadores sociológicos. Al inicio, se abordó desde la marcada influencia de las instituciones y hechos sociales sobre el comportamiento individual (Durkheim, 1997, p. 202). Después, se estudió desde la perspectiva del individuo, es decir, se puso el foco en conocer las regularidades de los comportamientos traducidos en intenciones y resultados de la acción individual (Weber, 1997). Asimismo, se ha entendido la acción social como el producto de estructuras materiales y de poder que predefinen el contenido de las ciencias en la historia, por lo que el análisis se enfoca en grandes colectivos, es decir, en los individuos como encarnación de su realidad económica y como representantes de una clase social (Marx, 1981). Otros acercamientos teóricos buscan entender la acción social como producto de las comunicaciones que se establecen en torno a la integración y diferenciación entre el sistema y su entorno (Luhmann, 1982). 
El individualismo metodológico weberiano intenta descubrir por qué los actores se comportaron de la manera en que lo hicieron para producir el fenómeno en cuestión; es decir: el fenómeno social es resultado de las acciones, actitudes y creencias individuales (Boudon, 1997, pp. 8-9), donde los conceptos de vida social como Estado, Iglesia y burocracia, están referidos a la acción de individuos (Albrow, 1990, p. 158). Es decir, no se trata de un individualismo ontológico, como si fuera una teoría como la del Rational Choice, con el supuesto de actores egoístas y maximizadores de beneficios, sino de un individualismo basado en el método interpretativo para explicar la realidad, sin supuestos de acción individuales, ya que eso es precisamente lo que se busca descubrir: el sentido de la acción individual. El presente artículo profundiza en la descripción del individualismo metodológico de Max Weber, que se centra en el sentido que otorga el individuo a su acción; esto se comprende mediante la construcción de un concepto racional de tipo histórico que utiliza distintas opciones de explicación con el fin de que sean amenazas a la validez de la hipótesis principal, al que también se llama tipos ideales (Kalberg, 2008).

El propósito de este artículo es presentar la propuesta metodológica weberiana de tipo ideal como una solución a este dilema acerca de los valores en la investigación de las ciencias sociales, donde se ofrece la separación de valores teóricos y prácticos como una solución a la disputa entre activismo e investigación. Para ello, el presente trabajo se divide en cinco partes. En la primera, se expone el contexto histórico de Max Weber y su obra. En la segunda parte, se presenta la propuesta weberiana de neutralidad valorativa; en la tercera se expone el análisis lógico o utilización de conceptos para la construcción de tipos ideales; en la cuarta parte se describe la manera en que el investigador interpreta la información empírica y la compara con los tipos ideales construidos. Finalmente, se hace una reflexión en torno a la pertinencia de la metodología weberiana para interpretar y comprender los fenómenos sociales contemporáneos.

\section{Breve contexto histórico de Max Weber y Su obra}

El término tipo ideal fue acuñado por Max Weber hace más de un siglo, sin embargo, ha sido poco utilizado como tal para interpretar la investigación empírica. Esto se debe a que los investigadores han optado más por el uso de los conceptos weberianos que al propio método en sí, debido a que Weber no dejó muy claro los pasos a seguir para construir el tipo ideal, por lo que se ha requerido estudiar más a fondo su significado (Swedberg, 2018). Parte de esta complejidad en la obra de Weber se debe a la evolución de sus ideas que puede apreciarse a lo largo de sus textos: mientras que al inicio se concentró más en desarrollar las disciplinas de historia y economía, al final de su vida se concentró más en el estudio de la sociología. Además, la malinterpretación de la obra de Weber, ya sea por errores de traducción o por falta de conocimiento profundo de la obra en sí, también ha contribuido a que su obra sea compleja (Rosenberg, 2016).

Por ejemplo, uno de los principales traductores y promotores de la obra de Weber en la lengua inglesa fue Talcott Parsons (1964), el mismo que también la sobreinterpretó, ya que afirmó que algunos conceptos buscaban formar una teoría general, mientras que sólo buscaban explicar una realidad histórica específica. Es decir, mientras Weber construyó un tipo ideal para explicar el rol de la burocracia en el contexto de la sociedad industrial, Parsons consideró que se trataba de una apología a la industrialización y burocratización, mientras que Weber sólo intentaba explicar una realidad histórica específica, incluso este último dejó testimonios de su temor de que la burocracia pudiera convertirse en una maquina deshumanizada del capitalismo, donde la imparcialidad del burócrata llegara al extremo de ser ciega a los fines de la sociedad, al tiempo que cumplía con su encargo burocrático de manera automática, a lo que Talcott Parsons, en la traducción al inglés de 
La ética protestante y el espiritu del capitalismo, llamó “jaula de hierro" (Weber, 1995; Weber, 1930, p. 123; Swedberg \& Agevall, 2016, p. 172). Este es uno de varios ejemplos donde Max Weber fue malinterpretado por sus traductores, estudiosos y críticos de sus obras.

Por su parte, las influencias en el pensamiento de Weber son varias, sin embargo, propongo el enfoque en tres personajes que aparecen constantemente en los distintos estudios sobre su vida y obra. No se pretende afirmar que son los únicos o los que más influyeron, ya que no es el objetivo de este artículo; ni siquiera estos tienen una jerarquía de influencia, sino que sólo se busca ofrecer un breve contexto histórico del pensamiento weberiano. Estos personajes son: Rickert, Dilthey, y Kant. En el caso de Rickert, neokantiano y un año mayor que Weber, destaca la influencia acerca de una concepción que parte de la pluralidad e imposibilidad de la jerarquización de valores. De hecho, Weber refiere a Rickert tanto para discutir con él como para una mayor comprensión de algunos conceptos que toma prestados de su filosofía, como la irracionalidad de la realidad, la relevancia de los valores, y la objetividad en las ciencias sociales. Cabe destacar que Rickert recibió influencia de su maestro Windelband, fundador de la Escuela Alemana del Sureste (Oakes, 1990, pp. 6-7).

Por su parte, Dilthey influyó en Weber en el entendimiento de las ciencias del espíritu como una cuestión intersubjetiva e histórica-cultural, producto de la experiencia vivida, más que una cuestión subjetivapsicológica sin una acción práctica (Ermarth, 1978). En el caso de la influencia de Kant, esta se da por la concepción del individuo como ser racional que toma decisiones libremente de acuerdo con una ética preestablecida, contrario a la idea de Hegel y Marx, quienes consideran al individuo como producto de las relaciones materiales de clase, donde sus decisiones se deben más al contexto natural-material, donde no se puede responsabilizar enteramente al individuo de las decisiones que toma, ya que son producto de una imposición material-estructural externa. Entonces, Weber marca distancia de Marx en este punto y toma la posición del individualismo metodológico que busca comprender la acción social del individuo y el sentido que da este a su acción (González, 1988, p. 26). No obstante, tanto Marx como Weber intentaron comprender los problemas de la existencia del capitalismo burgués, a través de un profundo análisis histórico del desarrollo del mundo occidental de la esclavitud, feudalismo y capitalismo (Löwith, 1993, p. 27).

Si algo marcó la obra y evolución del pensamiento de Max Weber fue la cuestión del rol de los valores en la investigación, inquietud que lo llevó a concluir, un año antes de su muerte, el texto de "la ciencia como vocación" (Ringer, 1997, p. 131), donde afirma que la ética del científico no es otra cosa más que la búsqueda de la verdad. Su interés por los valores fue influenciado por la Alemania en la que le tocó vivir, donde la Iglesia Católica tenía un fuerte control social y el nazismo empezaba a cobrar fuerza, lo que llevó a Weber a ser un activista en contra de la histeria nacionalista. Sin embargo, también defendió ideales de grupos ideológicos contrarios cuando lo consideró justo, por ejemplo, cuando algunos estudiantes socialistas de Múnich fueron maltratados por protestar por la absolución de un asesino, Weber habló en contra de este incidente, y se convirtió en objetivo de críticas por parte de la derecha alemana (Ringer, 1969, pp. 197-216). Este hecho destaca que cuando se defienden valores e ideales desde el activismo, hay que estar dispuestos a tener conflictos con las personas de las organizaciones que conforman diferentes ideologías, de lo contrario uno se podría convertir en presa de los grupos a los que pertenece, debido a los reclamos que se producen cuando la opinión va en contra de los intereses defendidos por dichos grupos. Para dar un ejemplo: si una persona pertenece a un partido político y se da cuenta de alguna injusticia, difícilmente confesará públicamente lo que realmente piensa del manejo partidario, ya que calcula que su opinión trastocará negativamente los intereses de dicha agrupación y, por lo tanto, recibirá reclamos de sus correligionarios.

De acuerdo con Radkau (2009, p. 4), biógrafo de Max Weber, las emociones no son contaminación del pensamiento, ya que ideas y emociones son inseparables. En este sentido, Weber fue marcado por la enfermedad de los nervios que padeció, así como por su vida familiar. En su infancia fue marcado por la educación de su padre, quien le sirvió como ejemplo de racionalidad y pragmatismo político, mientras que su madre representó un lado más afectivo, como el altruismo y el activismo protestante enfocado en causas sociales. En este dilema se encontró Weber desde su infancia, entre el prestigio de la fuerza del Estado Alemán 
y la búsqueda de causas justas desde el protestantismo (Cuneo, 1990, p. 86). Incluso, la profunda crisis nerviosa que experimentó Weber tras la muerte de su padre pudo haberse debido a la simbiosis paralizante de los dos lados de su personalidad influenciada por sus padres (Collins, 1986, p. 18). Curiosamente, la propuesta que hace Weber después de su recaída emocional es separar ambas esferas, por un lado, la racionalidad de la investigación científica y por el otro, el activismo práctico. Para ambas esferas propone que los valores sean elegidos libremente, tal vez pensando en la intransigencia de ciertas instituciones políticas de su tiempo, como el nazismo. Después de este breve e incompleto contexto de influencia a la obra de Max Weber, se procede al análisis de las principales ideas alrededor de la construcción de los tipos ideales como artificios metodológicos.

\section{La neutralidad Valorativa, como libertad de elección, en MaX Weber}

El núcleo conceptual weberiano de neutralidad valorativa es la distinción entre un razonamiento teórico - descriptivo y enfocado en cómo son los fenómenos- y otro práctico - prescriptivo y enfocado en cómo deben ser las cosas-. Basó la formación de este núcleo conceptual en Immanuel Kant, Heinrich Rickert, y en cierta medida fue inspirado también por Friederich Albert Lang. Weber buscó, con esta separación analítica del razonamiento teórico y práctico, acercarse a la verdad científica en las ciencias sociales, e insistió en la diversidad de valores y sobre el conflicto irreconciliable entre ellos. Refiriéndose a Friedrich Nietzsche y a Charles Baudelaire, Weber argumentó que "algo puede ser sagrado y no bello, también puede ser bello y malvado, así como verdadero y no bello, ni santo, ni bueno." (Ringer, 1997, p. 237). Weber incluso pensó a la verdad científica como un valor más en juego, ya que consideraba que, si una persona encontraba un cierto elemento de la vida teóricamente "interesante", "que vale la pena conocer", entonces, más o menos explícitamente, hacía una elección subjetiva a favor del valor de descubrir la verdad. Otras opciones son igualmente posibles, y Weber sabía que no se puede demostrar que uno debe elegir la verdad en lugar de algún otro valor (Bruun, 2007, p. 28).

El concepto de neutralidad valorativa (value-neutrality) es uno de los conceptos más polémicos y malinterpretados a la obra de Max Weber. Sus críticos afirman que es imposible eliminar los valores en el investigador social y que no es apto de ser neutral en sus juicios. Sin embargo, Weber no se refería a eso y afirmó que los valores juegan una función lógica en el método, no son en sí mismos el principio de validez cognoscitiva ni es necesario afirmar la absoluta universalidad de un sistema jerárquico de valores para poder aprobar la objetividad de los conceptos (Aguilar-Villanueva, 1988-1989). Weber propuso una forma de resolver la imposibilidad de la neutralidad valorativa hace más de un siglo, afirmando que la verdad científica (no la "Verdad universal"), requiere separar los valores teóricos de los valores prácticos del investigador, esto como un ideal al que el investigador debe intentar acercarse lo más posible; donde la clave de dicha neutralidad es la libertad de seleccionar valores teóricos para estudiarlos en contextos específicos, contrario a la idea de ser un fanático de ciertos valores. De acuerdo con la interpretación de Peón (1998) y Aguilar-Villanueva (1984), un investigador libre frente a los valores está obligado a transparentar su elección de valores, en cambio un investigador neutral frente a los valores reprime su apetito axiológico. Es decir, la neutralidad valorativa se refiere inminentemente a la libertad de elegir.

En este sentido, el investigador debe empezar por despojarse de todo interés práctico al momento de escribir o, al menos, reconocer claramente y hacer que otros reconozcan cuándo sus argumentos apelan a la razón y cuándo a sus emociones. Las opciones a investigar se construyen a partir de distintos valores que el investigador debe transparentar; lo que propone Weber es demostrar las consecuencias de la predominancia de ciertos valores teóricos sobre otros, no más que eso, porque los valores tienen una validez temporal y contextual. Cuando distingue entre juicio de valor teórico y práctico, este último se refiere a la actitud positiva o negativa de una persona sobre la base de un sentimiento sumamente individual, lo que puede nublar su raciocinio; en suma, es cuando se afirma que algo es bueno o malo con respecto a los valores absolutos de la 
persona que juzga. En cambio, el juicio de valor teórico, que utiliza Weber para construir sus tipos ideales, se basa en la observación y percepción del investigador desde una distancia al objeto de estudio y, por tanto, se encuentra en posición de modificar su punto de vista hacia él. El investigador establece que algo es adecuado de acuerdo con ciertos valores contextuales que está analizando, mas no toma posición personal en preferir ciertos valores sobre otros (Weber, 1995).

En su estudio clásico acerca de la ética protestante y el espíritu del capitalismo, por ejemplo, Max Weber demostró cómo estos dos conceptos estaban relacionados en la comprensión del fenómeno de expansión del capitalismo. Weber no era un defensor a ultranza del capitalismo, ni lo contrario, ni juzgaba que la ética protestante fuera buena o mala en sí, lo que hizo fue demostrar que la ética protestante era el concepto más adecuado para comprender la evolución del espíritu del capitalismo en sociedades industriales del siglo xix (Weber, 2003). Weber sugiere evitar la tentación de incorporar una mezcla no confesada de juicios objetivos y convicciones personales. Esto no significa que el investigador no pueda expresar sus valores personales y convicciones, sino que lo debe de hacer explícito y distinguir sus juicios de valor prácticos de los juicios de valor teóricos (Weber, 1995).

Esta tentación de mezclar juicios prácticos con teóricos puede darse más intensamente en los investigadores que son activistas políticos, ya que podrían sentir un cierto compromiso con la defensa de ciertos valores prácticos y rendirse a la pasión de sus convicciones al momento de hacer una investigación. Cabe señalar que no siempre es así: Weber fue un activista intelectual apasionado por la política alemana e internacional, y logró alcanzar una disciplina científica por medio del uso de valores teóricos para la construcción de tipos ideales. Un aspecto que Weber resaltaba entre sus estudiantes era el hecho de que no se le considerara a él como una personalidad, ya que este hecho iba en contra de la objetividad sencilla (libre de amor y odio en la investigación), donde las emociones del investigador se subordinadan a la materia científica, y cuando se admira una personalidad lo que se está invocando es lo contrario.

La diferenciación que hace Weber de las ciencias empíricas y las aproximaciones dogmático-valorativas se inspira en el contexto histórico que vivió, cuando surgió una tendencia de convertir a las universidades en centros de formación integral para el estudiante, donde no sólo se les formaba en el método científico sino que también se proclamaba la necesidad de fomentar ideologías críticas en los estudiantes. Weber estuvo en contra de esta tendencia porque afirmaba que la decisión acerca de qué valores preferir estaba reservado sólo para cada individuo y el docente no debía influenciar en nada esa decisión, porque la argumentación científica no obliga a nadie a tomar una decisión en la esfera de los valores prácticos. Añadió que si un docente desea fomentar ideologías políticas, que se vaya a un mercado o a un partido porque el aula no es el espacio para hacerlo. En este sentido, las ciencias empíricas son las que están basadas en la argumentación científica con la ayuda de valores teóricos, por el contrario, las aproximaciones dogmáticovalorativas son las que mezclan instrumentos científicos con activismo e ideologías políticas no confesadas en las investigaciones. En este contexto, es importante resaltar que Weber era un activista político apasionado y no le gustaba concentrarse exclusivamente en la investigación académica. Aún a pesar de ser un apasionado de ciertos valores prácticos, defendía la necesidad de que el investigador trabajara preponderantemente con valores teóricos, y si consideraba necesario argumentar sus investigaciones con valores prácticos, que los transparentara claramente (Weber, 1997, pp. 44-46).

A continuación, se presenta un ejemplo que se retomará más adelante con el fin de clarificar cada una de las fases de la construcción de tipos ideales. En lo que respecta a la neutralidad valorativa, el investigador primeramente piensa en un tema de investigación que le apasiona, ya sea por indignación o anhelo; supongamos que decide estudiar el patrón de decisión que predomina en la contratación de personal en un gobierno. Esta decisión representa una selección de valor, ya que se eligió un tema y se descartaron otros posibles, como el programa municipal de agua o el de recolección de basura. Ahora bien, ¿cómo se inicia la investigación para evitar un sesgo valorativo? Se va al mundo empírico a conocer cuáles son las posibles respuestas a nuestra pregunta, digamos que lo conseguimos por medio de entrevistas; o bien se puede acudir 
a la literatura para conocer qué experiencias existen en otros contextos. Tras una revisión de literatura y/o entrevistas a expertos o tomadores de decisiones en materia de contratación de personal, tenemos tres posibles patrones de decisión conocidos: burocrático, nueva gestión pública, y aparatos políticos. Ahora, estos tres posibles patrones de decisión se convierten en valores teóricos a estudiar. Después de aclarado el concepto de neutralidad-libertad valorativa y de entender la distinción entre juicios de valor prácticos y teóricos, a continuación, se profundiza en el método de tipo ideal.

\section{EL TIPO IDEAL WEBERIANO}

El tipo ideal es una acción racional, construida por el investigador, para estudiar cualquier acción social imaginable; Weber no puso límites a las variedades de tipo ideal: podrían ser una norma legal, un tipo de personalidad, un complejo institucional, o el Imperio Austrohúngaro. Estaba absolutamente alejado de sus intenciones el construir un inventario de tipos ideales. Lo fundamental en el tipo ideal de acción racional es el enfoque en los individuos y el sentido que estos le dan a la acción, para que en el agregado de acciones individuales se pueda determinar la probabilidad de ocurrencia de un cierto fenómeno social (Albrow, 1990; Ringer, 1997).

Para Weber las ciencias sociales deben ayudar a comprender -conocer el significado subjetivamente pensado por el actor- de la acción humana y su interpretación. Retoma el concepto de tipo ideal de Georg Jellinek y busca con este método evitar cometer el error de algunos historiadores que analizan el pasado con lentes actuales. Weber reconocía la calidad de los trabajos de Marx porque iluminaron zonas del conocimiento antes desconocidas, sin embargo, no estaba de acuerdo con que los factores materiales se volvieran absolutos y común denominador de las explicaciones causales. Si bien Weber reconocía que cada fenómeno cultural también está determinado económicamente, destacaba que no era sólo así (Weber, 1995).

De acuerdo con Aguilar-Villanueva (1988-1989), son tres las categorías analíticas centrales en Weber: acción, individuo y racionalidad. Su trabajo se focaliza en la identificación de la acción humana más relevante en el proceso de un acontecimiento histórico, en donde la interpretación racional cumple sólo una función instrumental en el método; es decir, Weber busca un concepto racional de la historia, no una historia racional. La conexión de la acción con arreglo a fines y valores no es directamente comprobable en el terreno empírico, pues la acción no es un dato, no está a la vista ni es un objeto observable, sino que es una relación del pensamiento, producto de la interpretación. Con el fin de esquematizar la propuesta metodológica weberiana, a continuación se presenta un resumen de sus tres elementos esenciales:

Tabla I

\begin{tabular}{|c|c|c|c|}
\hline $\begin{array}{l}\text { Juicio de } \\
\text { imputación } \\
\text { causal }\end{array}$ & Elemento & Descripción & Condición \\
\hline $\begin{array}{l}\text { Análisis de valor } \\
\text { teórico }\end{array}$ & Valores & $\begin{array}{l}\text { Selección de conceptos } \\
\text { y valores teóricos }\end{array}$ & Necesaria \\
\hline Análisis lógico & $\begin{array}{l}\text { Concepto } \\
\text { ideal }\end{array}$ & $\begin{array}{l}\text { Construcción de tipos } \\
\text { ideales, como acción } \\
\text { racional medios-fines }\end{array}$ & Necesaria \\
\hline $\begin{array}{l}\text { Sintesis de la } \\
\text { conexión } \\
\text { efectivamente } \\
\text { real }\end{array}$ & $\begin{array}{l}\text { Sentido } \\
\text { de la } \\
\text { acción }\end{array}$ & $\begin{array}{l}\text { Falseo de } \\
\text { contra-hipótesis y } \\
\text { determinación de la } \\
\text { hipótesis ganadora en } \\
\text { la realidad }\end{array}$ & Suficiente \\
\hline
\end{tabular}

Propuesta metodológica weberiana para construir tipos ideales Fuente: Cáñez-Cota (2011, p. 37). 
El análisis valorativo y conceptual -la selección del objeto de estudio- es el primer momento para la construcción de tipos ideales. Para Weber es inherente de la ciencia social comprender la acción para explicarla, no valorizarla como buena o mala, digna o indigna del ser humano. En las investigaciones sociales se encuentra que, consciente o inconscientemente, se trata el objeto de estudio con cargas valorativas. Esto es inevitable y por ello se hace necesario explicitar la carga valorativa de los conceptos y evaluarlos con respecto a sus mismos valores. El investigador no debe cargar los conceptos de valor sino ponerlos en evidencia para estudiar y evaluar la acción (Weber, 1997; Aguilar-Villanueva, 1988-1989).

El análisis de valor se refiere a la selección del objeto de estudio - de acuerdo con una relación de valor explícita- de los elementos más relevantes, dentro de la infinidad de factores que se pueden intentar explicar, de acuerdo con el interés histórico. Es decir, se selecciona un acontecimiento histórico con el objetivo de convertirlo en tipo ideal, donde las otras posibles explicaciones del mismo acontecimiento son tomadas en cuenta, también se construyen como tipo ideal quedan establecidas como contra-hipótesis (AguilarVillanueva, 1988-1989).

Desde la selección del objeto de estudio existe una carga valorativa porque se incluyen y excluyen elementos, se consideran algunos temas más importantes que otros. En las ciencias sociales la explicación causal de la acción social puede vagar por el infinito de posibilidades si no existe una relación de valor que sirva como referencia de la investigación. Lo que ha mantenido al método weberiano como un instrumento pertinente es que la relación de valor es histórica, no pretende defender valores universales, sino valores y visiones del mundo histórico-contextuales, así como las circunstancias de las acciones regulares concretas en un punto de la historia. Weber entiende por acción regular a la predominancia de acciones en un mismo sentido, aunque estas tengan excepciones (Kalberg, 2008). Los juicios de valor teóricos en Weber son los que permiten establecer las distintas relaciones causales de tipo ideal y conocer en la realidad empírica cuál de ellas, bajo esas circunstancias históricas específicas, es la que ocurre con mayor regularidad y qué resultados produce dicha acción. Sus críticos han afirmado que los juicios de valor producen un sesgo y limitación en su método: malinterpretan la propuesta metodológica weberiana porque la relación de valor es un punto de partida al rico y complejo pluralismo metodológico (Gil-Villegas, 2003). Ahora bien, una vez seleccionados los conceptos, por medio del análisis de valor, el segundo momento es establecer el análisis lógico de los conceptos ideales e hipótesis.

El análisis lógico se refiere a la construcción de los conceptos típico-ideales, los mismos que son una referencia para conocer el sentido que dan los actores a la acción continuada, es decir, la acción repetida y regular, no las acciones aisladas. Es común que se malinterprete el tipo ideal weberiano en el sentido de algo deseable, de algo ejemplar en el sentido de bien y deber ser (Morcillo y Weisz, 2016, pp. 17-19). Sin embargo, Weber no se refería a eso con el concepto de ideal, sino que se refería a la perfección lógica del concepto, no la axiología de la acción: alude al método, no a un proyecto de normatividad social. El tipo ideal es una idea, una abstracción de la realidad y supone que no suele presentarse, tal cual, en el mundo empírico, sino que es una construcción racional de los conceptos más relevantes que pueden explicar el fenómeno a investigar (Aguilar-Villanueva, 1988-1989).

Para que una investigación tenga un punto de partida adecuado se requiere definir con precisión y univocidad la realidad que se pretende explicar, es decir, es necesario marcar claramente los límites del concepto y que exista una única intencionalidad entre el actuar y el fin del concepto racional (AguilarVillanueva, 1988-1989). Weber pretende la formación de conceptos universales y no enunciados causales universales, por ello introduce la noción de probabilidad. No se trata con conceptos absolutos, sino que se trata de la probabilidad de ocurrencia de ciertos fenómenos; se busca comprender la predominancia de relaciones causales, no se intenta conocer una causalidad total y única. Es por ello que la metodología weberiana traduce sus resultados como probabilidad, no como algo universal y definitivo. Los resultados son probabilidades porque se ajustan al marco de medios, fines, valores, circunstancias y resultados de la acción histórica: es probabilidad porque es contextual (Aguilar-Villanueva, 1988-1989). 
Es necesario definir el tipo ideal con precisión y univocidad, no en la definición tradicional de diccionario sino en una conexión genética (esta última como la estructura intencional propia de una acción histórica). La precisión debe entenderse como esfuerzo analítico para encontrar una cadena lógica de la acción y univocidad como la no contradicción de los elementos que componen la acción; precisión y univocidad hacen que los tipos ideales sean extraños a la realidad empírica. Esta es la genialidad de Weber al momento de construir su método de tipo ideal, ya que en la realidad no se pueden separar las acciones si estas son contradictorias. Por ello construye la acción racional de tipo ideal, para lograr precisión y univocidad en el terreno analítico y así estudiar los fenómenos sociales como probabilidades de ocurrencia de una acción (Aguilar-Villanueva, 1988-1989). La definición del concepto típico ideal acorde con las condiciones históricas específicas es una parte central en la metodología weberiana, lo cual no se ha comprendido adecuadamente, ya que algunos críticos han entendido los conceptos como universales, mismo que ha llevado a la malinterpretación de su método. Los críticos han reclamado que pretendió relacionar causalmente el protestantismo con el capitalismo: esta confusión de lectura de conceptos es igual de perjudicial que la construcción de la conceptualización del estudio. Weber construyó tipos ideales con base en la historia de un contexto en específico, a partir de ahí relacionó ética con espíritu, esa es la conexión significativa y no el protestantismo y el capitalismo como lo dicen sus críticos (Gil-Villegas, 2003). Este ejemplo muestra la relevancia que tiene definir los conceptos con la mayor claridad posible.

En el mundo empírico no se encontrará el blanco y negro como se expresan en los tipos ideales que construye el investigador, se tendrán distintas escalas de grises que se parecerán, en mayor o menor medida, a algún tipo ideal, donde la regularidad de las acciones será la que otorga el predominio de uno sobre otro (Aguilar-Villanueva, 1988-1989). El tipo ideal no suele verse, en su tipo puro, en la realidad, ya que es una construcción conceptual-analítica, es un medio para lograr causalidad no para ver el mundo como el tipo ideal, ya que la realidad es más compleja. Max Weber pretende construir conceptos universales transformados en modelos y no pretende establecer enunciados causales totales, y es por ello que introduce el término de probabilidad. La construcción del tipo ideal requiere trabajar sobre tres elementos fundamentales del concepto de acción: racionalidad, consecuencias y arreglo a valores. La racionalidad se entiende como el conjunto de medios de los que dispone el actor y los fines que busca obtener o generar con la acción; las consecuencias como los efectos concretos producidos; y el arreglo a valores, como el referente valorativo del actor que determina, en última instancia, el sentido de la acción (Aguilar-Villanueva, 1988-1989). En otras palabras, por racionalidad de una acción se entiende la selección y empleo de los medios que son idóneos, en razón de su eficacia, para la realización de un fin determinado o, dicho a la inversa, en razón del fin buscado por el actor, es consecuencia el empleo de esos medios. No existe otra manera de establecer y caracterizar la racionalidad que no sea con la regularidad empírica de su ocurrencia. Una acción racional requiere que exista un propósito deliberado por parte del actor para alcanzar el fin (Aguilar-Villanueva, 1988-1989). Es importante destacar que no existe una racionalidad única, sino que existen distintas racionalidades, de acuerdo con el tipo ideal que se construya.

La hipótesis en el método weberiano se desprende del tipo ideal y es un acto de selección de una línea causal y exclusión de otros cursos posibles. Esta se acompaña de contra-hipótesis que amenazan la veracidad de la hipótesis principal. El tipo ideal es útil para distinguir las acciones sociales, ya que este se aleja de la realidad para poder observar, analíticamente, la acción que se lleva a cabo con mayor regularidad (Weber, 1997). Las acciones y relaciones descartadas de calidad causal, importantes en el proceso de generación del hecho histórico, quedan entonces como amenazas empíricas de la verdad de la hipótesis principal. Quedan como hipótesis alternativas o contra-hipótesis, se vuelven elementos de falsación de la hipótesis preferida si llegan a contar con la prueba empírica. Esta multiplicidad de opciones, al observar la realidad histórica, es lo que da potencia al método de tipos ideales (Aguilar-Villanueva, 1988-1989). Retomando el ejemplo del patrón de decisión que predomina en el proceso de contratación de personal de un gobierno, en este segundo momento de análisis lógico de construcción de tipos ideales, se procede a construir cada uno de los 
tres conceptos seleccionados como acción racional. Esto es, exagerar analíticamente la acción para convertirla en tipo ideal puro -en función de medios, fines y consecuencias de la acción-, el cual no suele ocurrir, en su tipo puro, en la realidad.

En el caso del patrón de decisión burocrático, se tiene que el medio utilizado por el actor es el apego a la normatividad, los fines de su acción van encaminados a lograr una imparcialidad impersonal en su rutina laboral, mientras que las consecuencias de los actos de los burócratas producen un sistema meritocrático basado en la ley. En lo que respecta al patrón de nueva gestión pública, los medios que utiliza el actor son volubles de acuerdo con la satisfacción del cliente; los fines del burócrata, en este caso conceptualizado como agente o gerente público, están acordes a la eficacia de alcanzar los objetivos propuestos en una planeación estratégica, mientras que las consecuencias producidas refuerzan un sistema flexible, basado más en el logro de resultados que en el seguimiento a procedimientos. En relación con el patrón de aparatos políticos, los medios que utiliza el actor son el apego a las reglas legales; los fines del agente político están en función de ganar posiciones de poder para el grupo dominante en turno, mientras que las consecuencias de los actos de estos agentes políticos producen un sistema botín. En este ejemplo se aprecia la existencia de tres tipos de racionalidades posibles para la contratación de personal en los gobiernos latinoamericanos contemporáneos: un burócrata en un sistema orientado a la meritocracia, un gerente público en un sistema orientado a resultados, y un agente político en un sistema orientado a la ganancia y permanencia de posiciones de poder.

Luego de haber explicado el análisis de valor teórico para seleccionar el objeto de estudio, como primer elemento en la construcción de tipos ideales; y luego de haber presentado, como segundo elemento, el análisis lógico para la construcción de tipos ideales, a continuación se expone el tercer y último elemento.

\section{JUICIO DE IMPUTACIÓN CAUSAL: SÍNTESIS DE LA CONEXIÓN EFECTIVAMENTE REAL}

Lo que pretende el método weberiano es el juicio de imputación causal, que es producto de la síntesis de la conexión efectivamente real, es decir, el grado de concordancia entre la lógica de la racionalidad del tipo ideal y lo que se encuentra en el terreno empírico. Es preciso señalar que cuando se tienen los componentes de una interpretación causal correcta de una acción es cuando el desarrollo externo (medios) y el sentido (fines) han sido conocidos con certeza y comprendidos en su conexión lógica (Aguilar-Villanueva, 1988-1989). También cabe señalar que cuando los tipos ideales no pasan la prueba empírica es porque no presentan un nexo directo y lógico entre los medios utilizados por el actor y los fines que busca con su sentido de la acción, así como las consecuencias obtenidas. El tipo ideal que pasa la prueba empírica es el que establece una relación precisa y unívoca entre los medios, fines y consecuencias de la acción, representando, en mayor medida, lo que acontece en la realidad estudiada. Es decir que no existe otra explicación lógica para ese fenómeno en ese contexto específico (Aguilar-Villanueva, 1988-1989).

El establecimiento de la imputación causal probada exige ser planteada en una escala de certeza. La certidumbre de la seguridad en la prueba de hipótesis no podrá ser más que relativa, por eso Weber propone que toda causalidad es una probabilidad a ocurrir en la realidad social. Depende del conocimiento, recabado empíricamente, que una de las varias hipótesis puestas a prueba logra alcanzar mayor validez: la que cuente con mayor información que la sustente, será la que favorece causalmente la relación de conceptos que se propone. Esta hipótesis causal preferida es el punto crucial de la investigación y no puede determinarse más que comparativamente, es decir, cuando se contrasta el grado de ocurrencia que tiene la hipótesis principal contra las hipótesis alternativas, se está comparando la validez de los tipos ideales (AguilarVillanueva, 1988-1989). Es preciso destacar que en el análisis empírico aparecen mezclados rasgos de cada tipo ideal, pero el predominio de uno sobre otro es lo que da el sentido de la acción. Medios, fines, valores y consecuencias afirman la síntesis de la conexión efectivamente real (Aguilar-Villanueva, 1988-1989). Además de las estadísticas y hechos históricos documentados, considero que un instrumento adicional para conocer el sentido de la acción y estar en posición de hacer el juicio de imputación causal, es la entrevista, como la 
que se utiliza aquí para ejemplificar una manera de recabar datos empíricos y contrastarlos con el tipo ideal construido.

La validez de la explicación es relativa al número de contrastaciones o comparaciones que, con pertinencia cognoscitiva y para fines de la falsación de la hipótesis, se pueden establecer y realizar entre conjuntos alternativos de condiciones, de acuerdo al estado de conocimiento disponible. En este caso, que las estadísticas, los datos históricos recopilados y construidos, así como las encuestas y entrevistas deben ir en un mismo sentido para que una hipótesis prevalezca sobre las otras, que reclaman de igual manera su predominancia de regularidad empírica (Weber, 1964).

Los criterios centrales para recopilar los datos empíricos son la confiabilidad y la validez, con el fin de que las respuestas no varíen demasiado y se logre la saturación de la respuesta en un mismo sentido. Para obtener la confiabilidad en el caso de la entrevista, la estrategia es contrastar las respuestas de varios actores involucrados y relacionados con el fenómeno que se estudia, con el fin de contar con amplitud en las posibles respuestas. Además, la codificación de los resultados de las entrevistas permite la posibilidad de que estas se saturen en un sentido; es decir, la codificación de acuerdo con los tipos ideales construidos, que abarquen las posibles respuestas, permite tener un mapa lo suficientemente claro para que los resultados, en el caso de contar con saturación en las respuestas en un mismo sentido, cuenten con validez. En lo relacionado con la recopilación de la información empírica, en la codificación de las respuestas de los distintos instrumentos utilizados se toma el criterio de coincidencia como la característica para aglutinar las respuestas que van en un mismo sentido. Lo que interesa en la codificación de las respuestas es la información que comunican los actores involucrados en el fenómeno a explicar, debido a que cuando se analizan decisiones se hace más explícita la acción (Cortés, 2008, pp. 45-46). La coincidencia de la acción en un mismo sentido referido a un tipo ideal es lo que permite hacer la conexión efectivamente real de causalidad histórica-contextual.

En este último punto, se pone énfasis en obtener dos cosas: por un lado, los medios que los actores utilizan para tomar la decisión y, por otro, los fines y valores, es decir, el sentido de la acción (Aguilar-Villanueva, 1988-1989). Para ejemplificar con el instrumento de la entrevista, se aplica un cuestionario a varios actores que toman decisiones y se colocan las respuestas de acuerdo a su cercanía con el tipo ideal correspondiente. Dichos cuestionamientos se basan en conocer el cómo - medios que dispone el actor-y el por qué -fines de la acción- de la toma de decisiones, enfatizando en qué valor y motivación invoca cada quien -arreglo a valores-, así como en las consecuencias producidas por sus decisiones.

Retomemos nuestro ejemplo, en el supuesto de que el fenómeno a explicar sea el patrón de decisión que predomina en la contratación de personal en un gobierno. Con base en la realidad y en la teoría se construyen tres probables explicaciones como tipos ideales: burocrático, nueva gestión pública y aparatos políticos. Se procede a entrevistar a los encargados de contratar el personal y se les pregunta cómo y por qué toman la decisión de contratar. Si la mayoría de las respuestas en las que los medios, fines, valores y consecuencias van en el sentido de contratación con base en las credenciales y méritos de las personas, así como en la perspectiva de una carrera de servicio civil, el patrón de decisión que predomina será el burocrático. En cambio, si la mayoría de las respuestas afirman que la contratación se da teniendo en cuenta la actitud de la persona, el rendimiento esperado y la flexibilidad de su contratación con base en resultados, el patrón de decisión que predomina es el de nueva gestión pública. Por su parte, si la mayoría de los entrevistados responden que las contrataciones están en función del grado de parentesco o afinidad política de los dirigentes del sindicato o de los dirigentes gubernamentales, entonces el patrón de decisión es de aparatos políticos. Cabe señalar que durante las entrevistas se pueden mezclar los tipos ideales; sin embargo, siempre habrá un valor que predomina sobre los otros. Por ejemplo, en un caso supuesto en que se contrata a un pariente del dirigente sindical que tiene maestría y los documentos necesarios para el puesto, habría que analizar las otras contrataciones para obtener las regularidades de la acción. También sería necesario conocer cuáles fueron los motivos de la contratación, si fue por sus credenciales por su parentesco e influencia personal: uno debe pesar más que el otro, y eso es lo que el entrevistador debería buscar, para conocer el sentido de la acción. 
El análisis anterior se puede fortalecer con datos estadísticos y con análisis de documentos oficiales, además pueden incorporarse más construcciones de tipo ideal como contra-hipótesis. Sin embargo, el método mismo dará luz si los conceptos típico-ideales son suficientes, porque la acción que realmente ocurre tiene que ser explicada e interpretada por un tipo ideal con precisión y univocidad, de lo contrario la construcción metodológica de los tipos ideales sería insuficiente. La potencia explicativa del método de tipo ideal es que estos se construyen de la realidad histórica contextual, es decir, la interpretación de la acción es contundente porque el punto de partida de los conceptos de acción racional es la realidad en la que se encuentra el fenómeno a estudiar (Aguilar-Villanueva, 1988-1989). En caso de que en las entrevistas surja una hipótesis no considerada, se debe convertir una acción racional para ella y considerarla en las entrevistas posteriores.

Antes de pasar a las reflexiones finales, es necesario enfatizar que la explicación en Weber es críticamente autoconsciente de que no puede ofrecer una definición universal. Lo que ofrece es una explicación empíricamente adecuada al marco de las condiciones culturales, históricas y conceptuales en las que se inscribe y enuncia, pero no propone una explicación irrefutable o irrefrenable; la explicación es relativa, pero no arbitraria ni injustificable (Aguilar-Villanueva, 1988-1989).

\section{REFLEXIÓN FINAL}

En esta cuarta y última parte se resumen las fortalezas y críticas del método de Max Weber, para luego concluir con un par de reflexiones. Las fortalezas del método de tipo ideal weberiano se pueden resumir en tres. La primera se refiere a la racionalidad de la acción como instrumento del método, el observar y estudiar un fenómeno social en el vacío no lleva a un resultado metodológico sólido; la acción racional como método es una fortaleza fundamental ya que permite comparar la realidad con el tipo ideal y concluir el estudio con disciplina científica (Aguilar-Villanueva, 1988-1989).

La segunda fortaleza del método weberiano es la exploración del sentido de la acción. El actuar es difícil de aprehender debido a que existen múltiples motivaciones y sentimientos que se cruzan en la realidad; el enfocarse en el sentido de la acción permite establecer relaciones causales confiables porque se evalúa la acción con base en el valor que invocó el actor. La tercera fortaleza del método weberiano es el enfoque en las regularidades de la acción, la afirmación de que una acción es la que efectivamente se llevó a cabo es una afirmación difícil de mantener científicamente, para ello Weber introduce el término probabilidad. De esta manera, la afirmación no es absoluta, sino que se afirma como la probabilidad de que la acción ocurra con regularidad; es decir, que una explicación o hipótesis sea más probable que otras, en cierto contexto (AguilarVillanueva, 1988-1989).

Las críticas más comunes al método de tipo ideal weberiano se pueden resumir también en tres. La primera es la orientación hacia el sentido de la acción: los críticos niegan la posibilidad de que se tome como base a las motivaciones de los individuos porque estas son inestables (Durkheim, 1997, p. 187). Por otro lado, algunos críticos de Weber consideran que es parte de una revolución pasiva en contra del movimiento laboral socialista, ya que cuando analizó las relaciones económicas las pensó en un contexto de capitalismo desarrollado, como el empleado "bien pagado" de Estados Unidos, el mismo que retoma en su totalidad, afirman sus críticos, las formas burguesas, abandonando la posibilidad de un contexto de empleados mal remunerados (Rehmann, 2013, p. 9). Ante esto, considero que Weber desarrolló un tipo ideal de acción racional del agente del capitalismo en su estado puro, y a partir de ahí analizó la situación alemana, que se acerca a ese estado puro. En cambio, para la realidad latinoamericana se podría pensar en construir otro actor racional de tipo ideal que esté más apegado a prácticas capitalistas no desarrolladas, y poder acuñar, lógicamente, una acción social que sea predominante en otros contextos. Es decir, si dejamos de lado el contexto alemán de aquella época con un empleado promedio bien remunerado, y nos trasladamos al presente latinoamericano, la construcción de tipo ideal de empleado sería, probablemente, el de uno precarizado. 
La segunda crítica al método weberiano cuestiona la utilidad de que el método incluya valores (Durkheim, 1997, p. 68). Sin embargo, considero que son las mismas tradiciones y valores los que otorgan la calidad científica, ya que se toman los valores y tradiciones del momento histórico que se está analizando y se elevan al nivel de conceptos. El método weberiano explícita los valores y trabaja con ellos, no los esconde ni los jerarquiza (Weber, 1997, pp. 66-78). Una contrarréplica de sus críticos es que el individualismo libre de valores no es congruente cuando Weber piensa en el actor racional calculador, el cual es nada neutral, ya que básicamente es el agente del capitalismo: Weber no pensó en otros individuos no calculadores como pueden ser los miembros de sindicatos, que tienen una tradición de cooperativismo y asambleísmo (Allen, 2004, p. 80). Sin embargo, Weber refería que, aún en este mundo desencantado y burocrático, hay espacio para que cada individuo otorgue sentido a sus acciones hacia una causa o valor (Lassman \& Speirs 1994, p. xxiii). Considero que esta crítica al actor racional weberiano como acotado a la modernidad, calculadora e individualista, pudiera tener una fecha de caducidad si los individuos y la organización humana logran mudar, totalmente, su egoísmo individual y dan un paso a un estado colectivista y altruista, como el comunismo que propone Marx. Creo que, en esa situación histórica, probablemente el actor racional calculador que supone Weber se vería rebasado. Como agenda futura de investigación sería pertinente saber en qué grado la metodología weberiana sería útil en un contexto humano sin egoísmo y calculabilidad individual (tal vez dejaría de serlo).

La tercera crítica al método weberiano cuestiona la acción racional. Señala que las personas actúan por pragmatismo, que se encuentran en un ambiente de restricciones y es difícil conocer la acción real con base en una acción conceptual (Merton, 2002). Es cierto que existe un amplio consenso sobre que en el mundo real se desarrollan una serie de acciones que son de racionalidad limitada (Simon, 1984), sin embargo, los individuos tienen un sentido de la acción y se apegan a un valor al momento de tomar una decisión. Asimismo, los medios, fines y consecuencias de la acción son precisas y unívocas con alguno de los tipos ideales construidos. La confusión de los críticos de Weber está entre la acción racional como instrumento del método y la acción efectivamente realizada, que sin duda es de racionalidad limitada (Gil-Villegas, 2003).

Como primera reflexión final, la metodología Weberiana construye y propone los supuestos de acción para cada acción histórica, las cuales son contrastadas con la realidad en la que los supuestos de cada posible interpretación se ponen en evidencia y no se establece el sesgo de supuestos conductuales desde el inicio. La potencia del método de Weber es que no fuerza la realidad para que la información se ajuste a los modelos creados. Estos se generan a partir de la información empírica histórica y después se va a la realidad específica para comprobar cuál es la acción que tiene mayor regularidad de ocurrencia. Lo contrario sucede cuando se crea un modelo analítico, sin relación con el contexto que se estudia, en el que se buscan las variables que mayor se acomoden a la existencia de datos, sin tomar en cuenta el contexto histórico de las regularidades. $\mathrm{O}$ incluso se busca que los resultados empíricos se ajusten a cierta ideología preferida por el investigador.

Como segunda y última reflexión: una investigación científica no puede alcanzar la generalización de sus conclusiones con un sólo caso de estudio analizado; ni siquiera puede hacerlo con varios casos. Sin embargo, tampoco se puede afirmar que las conclusiones no sean válidas para otros contextos con circunstancias similares. La generalización se puede alcanzar incluyendo más casos en los que la repetición de los hallazgos y conclusiones en un mismo sentido permitan una conclusión con un mayor grado de presencia. Se puede concluir en la causalidad del predominio de una cierta acción histórica porque se profundiza en la relación causa-efecto y en la explicación de los elementos que la incluyen. En resumen, con base en lo anteriormente expuesto considero que el método de Max Weber es potente para la interpretación de las acciones sociales y que sigue vigente debido a su capacidad de evaluar empíricamente los hechos sociales en un momento histórico determinado, bajo una óptica de probabilidad que permite contrastar diversas hipótesis con lo que sucede en la realidad social, sin generar axiomas universales. Por el contrario, lo que propone son explicaciones que siempre podrán ser reinterpretadas cuando cambien las condiciones del contexto analizado. En cuanto al dilema de activismo e investigación, considero que es posible, siempre y cuando sea por separado. 
Contrario a lo que se piensa, Weber de ninguna manera consideró inapropiado tomar inspiración científica del activismo apasionado, y viceversa. Lo que no toleraba era la combinación dogmática y acrítica de ambas esferas (Mommsen, 1989, p. 8).

Weber fue un apasionado de la política práctica y un defensor acérrimo de la no inclusión de valores prácticos en las ciencias sociales. En cambio, propuso la utilización de valores teóricos como artificio metodológico para crear tipos ideales. Así fue como creó el concepto de burocracia, tema que ha sido malinterpretado por muchos: los críticos argumentaron que la burocracia tenía muchas fallas y que no era un ideal a seguir; pero Weber nunca defendió a la burocracia como una idea a seguir, sino como un concepto que definía los patrones culturales de una época. De hecho, cuando Weber mostró claramente sus valores prácticos con respecto a la burocracia lo hizo para advertir que, llevada al extremo, esta podía llegar a convertirse en una maquinaria deshumanizada. Pero esa preocupación práctica no afectó en absoluto el rigor teórico-metodológico de Weber.

La manera weberiana de investigar las ciencias sociales es un estilo que no deberían olvidar las nuevas generaciones de investigadores sociales. En ese sentido, considero que, si Weber leyera estas palabras, seguramente nos exigiría exhortar a los investigadores para que lo sigan críticamente y que no lo vean como un profeta, ya que esto podría nublar sus juicios teóricos. La tarea que pide Weber es guardar las pasiones al momento de redactar las investigaciones. Menuda tarea, ¿será posible? Pues él la logró y nos dejó un método para intentarlo: el tipo ideal.

\section{Bibliografía}

Aguilar-Villanueva, L. (1984). El programa teórico político de Max Weber. En F. Galván y L. Cervantes (Ed.), Política y desilusión lecturas sobre Weber. México: Universidad Autónoma Metropolitana.

Aguilar-Villanueva, L. (1988-1989) Weber: la idea de ciencia social [volumen I y II]. México: Editorial Porrúa.

Albrow, M. (1990). Max Weber's construction of social theory. New York: St. Martin's Press.

Allen, K. (2004). Max Weber. A critical introduction. London: Pluto Press.

Boudon, R. (1997). Thepresent relevance of Max Weber's wertrationalitiit (value rationality). En P. Koslowski (Eds.), Methodology of the social sciences, ethics, and economics in the newer historical school from Max Weber and Rickert to Sombart and Rothacker (pp. 3-31). Heidelberg: Springer.

Bruun, H. (2007). Science, values and politics in Max Weber's methodology. Hampshire: Ashgate.

Cáñez-Cota, A. (2011). Patrones de decisión en la acción gubernamental municipal en México: el caso de Hermosillo, Sonora (Tesis de maestría inédita). Centro de Investigación y Docencia Económicas, México. Recuperado de: h ttp://repositorio-digital.cide.edu/handle/11651/1948

Collins, R. (1986). Max Weber. A skeleton key. Beverly Hills: SAGE Publications.

Cortés, F. (2008). Algunos aspectos de la controversia entre la investigación cualitativa y la investigación cuantitativa. En F. Cortés, A. Escobar, y M. González (Ed.). Método cientifico y política social. A propósito de las evaluaciones cualitativas de programas sociales (pp. 27-58). México: El Colegio de México.

Cuneo, M. (1990). Values and meaning: Max Weber's approach to the idea of ultimate reality and meaning. Ultimate Reality and Meaning, 13(2), 84-95.

Durkheim, E. (1997) Las reglas del método sociológico. México: Fondo de Cultura Económica.

Ermarth, M. (1978). Wilhelm Dilthey. The critique of historical reason. Chicago: University of Chicago Press.

Gil-Villegas, F. (2003) Introducción del editor. En M. Weber, La ética protestantey el espíritu del capitalismo (pp. 9-50). México: Fondo de Cultura Económica.

González, J. (1988). Las herencias de Kant y de Goethe en el pensamiento de Max Weber. Reis: Revista Española de Investigaciones Sociológicas, 43(3), 23-42.

Kalberg, S. (2008) Max Weber, principales dimensiones de su obra. Buenos Aires: Prometeo. 
Lassman, P. \& Speirs, R. (1994). Weber political writings. New York: Cambridge University Press.

Löwith, K. (1993). Max Weber and Karl Marx. London: Routledge.

Luhmann, N. (1982). Organizacion y decisión. Autopoiesis, acción y entendimiento comunicativo. Barcelona: Anthropos.

Marx, K. (1981). El capital. Crítica de la economía politica. Libro I: El proceso de producción del capital. Madrid: Siglo XXI Editores.

Merton, R. (2002) Teoría y estructura sociales. Buenos Aires: Fondo de Cultura Económica.

Mommsen, W. (1989). The political and social theory of Max Weber. Collected essays. Chicago: The University of Chicago Press.

Morcillo Laiz, Á. y Weisz, E. (2016). La relevancia para Iberoamérica de las interpretaciones sobre Max Weber. En A. Morcillo, y E. Weisz, (Ed.), Max Weber en Iberoamérica. Nuevas interpretaciones, estudios empiricos y recepción. México: Fondo de Cultura Económica-CIDE.

Oakes, G. (1990). Weber and Rickert. Concept formation in the cultural sciences. Cambridge: The MIT Press

Parsons, T. (1964). Introduction. En M. Weber, The theory of social and economic organization (pp. 3-86) (Trans. Talcott Parsons). New York: The Free Press

Peón, C. (1998). Max Weber en América Latina: su recepción temprana y algunas claves de lectura. En L. Aguilar, C. E. Peón y J. Pinto (Ed.), La politica como respuesta al desencantamiento del mundo. El aporte de Max Weber al debate democrático. Buenos Aires: EUDEBA.

Radkau, J. (2009). Max Weber. A biography. Cambridge: Polity Press.

Rehmann, J. (2013). Max Weber: Modernisation as passive revolution. A Gramscian analysis (Trans. Max Henniger). Leiden: Brill.

Ringer, F. (1969). Thedecline of the German mandarines. TheGerman academic community, 1890-1933. Cambridge: Harvard University Press.

Ringer, F. (1997). Max Weber's methodology. The unification of the cultural and social sciences. Cambridge: Harvard University Press.

Riso, W. (1951). El poder del pensamiento flexible: de una menta rígida a una mente libre y abierta al cambio. Bogotá: Grupo Editorial Norma.

Rosenberg, M. (2016). The conceptual articulation of the reality of life: Max Weber's theoretical constitution of sociological ideal types. Journal of Classical Sociology, 16(1), 84-101.

Simon, H. (1984). El comportamiento administrativo, estudio de los procesos decisorios en la organización administrativa. Buenos Aires: Aguilar.

Swedberg, R. (2018). How to use Max Weber's ideal type in sociological analysis. Journal of Classical Sociology, 18(3), 181-196.

Swedberg, R. \& Agevall, O. (2016). The Max Weber dictionary. Key words and central concepts. Stanford: Stanford University Press.

Weber, M. (1930). The protestant ethic and the spirit of capitalism. New York: Routledge.

Weber, M. (1964). Economía y sociedad. México: Fondo de Cultura Económica.

Weber, M. (1995). Biografía de Max Weber. México: Fondo de Cultura Económica.

Weber, M. (1997). Ensayo sobre metodología sociológica. Buenos Aires: Amorrortu.

Weber, M. (2003). La ética protestante y el espiritu del capitalismo. México: Fondo de Cultura Económica. 